\title{
Eye care for your patients with diabetes - screening, diagnosis and treatment options
}

\section{Soins oculovisuels pour vos patients diabétiques - dépistage, diagnostic et options de traitement}

\author{
BY / PAR MARGARET BEATTY RN, (inf.), MHSc, CHE
}

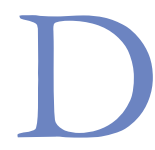
iabetic retinopathy is the most common complication of diabetes and cause of legal blindness in aging Canadians. It is estimated two million Canadians (i.e. almost all people diagnosed with diabetes) have some form of diabetic retinopathy. As optometrists, you play a vital role in the care of your patients who have diabetes or pre-diabetes to screen, diagnose and prevent the onset and/ or progression of retinopathy. Type 1 and type 2 diabetes both carry risks for the development of proliferative retinopathy; with hyperglycemia being the single most important risk factor. The prevalence is $23 \%$ for people with type 1 diabetes, 14\% for people with type 2 diabetes on insulin therapy, and 3\% in those receiving oral anti-diabetes medications. The Canadian Diabetes Association actively supports clinical research to establish scientific evidence to address diabetic retinopathy. The following information represents the results of that research.
Diabetic retinopathy, defined in the CDA's 2008 Clinical Practice Guidelines, may be classified in three main forms:

1.) macular edema, diffuse or focal vascular leakage at the macula;

2.) non-proliferative diabetic retinopathy: progressive accumulation of blood vessel change that includes micro aneurysms, intraretinal hemorrhage, vascular tortuosity and vascular malformation;

3.) retinal capillary closure, a form of vascular change, a potentially blinding complication of diabetes.

\section{Recommendations}

In individuals $\geq 15$ years of age with type 1 diabetes, screening and evaluation for retinopathy by an expert professional should be performed annually starting at five years after the onset of diabetes.

In individuals with type 2 diabetes, screening and evaluation for diabetic retinopathy by an expert professional should be performed

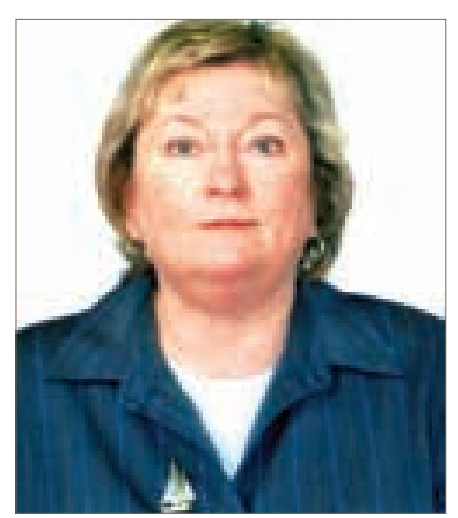

Margaret Beatty is the Vice President of Research, Professional Education and Government Affairs, at the Canadian Diabetes Association

at the time of diagnosis of diabetes. The interval for follow-up assessments should be tailored to the severity of the retinopathy. In those with no or minimal retinopathy, the recommended interval is one to two years.

Screening for diabetic retinopathy should be performed by experienced professionals, either in person or through interpretation of retinal photographs taken through dilated pupils. 
To prevent onset and delay the progression of diabetic retinopathy, people with diabetes should be treated to achieve optimal control of blood glucose and BP. People with abnormal lipids should be considered at high risk for retinopathy.

Patients with sight-threatening diabetic retinopathy should be assessed by a general ophthamologist or retinal specialist. Consider laser therapy and/or vitrectomy and/or pharmacologic intervention.

Refer visually disabled people to low-vision evaluation and rehabiliatation.

Optometrists, the first line of eye care clinicians, can make a difference for people with diabetes and their eye care. We hope this information and the full chapter on retinopathy in the 2008 Clinical Practice Guidelines (found at diabetes.ca) will help you broaden delivery of care to your patients with diabetes.

†Canadian Diabetes Association Clinical Practice Guidelines Expert Committee. Canadian Diabetes Association 2008 Clinical Practice Guidelines for the Prevention and Management of Diabetes in Canada. Can. J Diabetes. 2008; 32 (suppl. 1): S134-139.
L a rétinopathie diabétique est la complication la plus courante du diabète et la cause la plus fréquente des cas de cécité légale chez les canadiens âgés. On estime que deux millions de canadiens (c.-à-d. presque toutes les personnes ayant reçu un diagnostic de diabète) ont une forme ou l'autre de rétinopathie diabétique. En tant qu'optométristes, vous jouez un rôle essentiel dans les soins de vos patients diabétiques et prédiabétiques, en dépistant, diagnostiquant et prévenant l'apparition ou la progression de la rétinopathie. Le diabète de type 1 et le diabète de type 2 peuvent tous deux causer le développement de la rétinopathie proliférante, l'hyperglycémie étant le facteur de risque le plus important. Le taux de prévalence est de $23 \%$ chez les personnes atteintes de diabète de type 1 , de $14 \%$ chez les personnes atteintes de diabète de type 2 qui reçoivent une insulinothérapie et de $3 \%$ chez les personnes qui prennent des antidiabétiques oraux. L'Association canadienne du diabète appuie activement la recherche clinique afin d'établir des observations scientifiques qui permettront de faire face à la rétinopathie diabétique. Les renseignements qui suivent représentent les résultats de cette recherche.

La rétinopathie diabétique, définie dans les Lignes directrices de pratique clinique 2008 de l'ACD, comporte trois formes principales : 1.) l'œdème maculaire, qui résulte d'une fuite vasculaire diffuse ou focale au niveau de la macula;

2.) la rétinopathie diabétique non proliférante : l'accumulation progressive d'altérations des vaisseaux sanguins, telles que microanévrismes, hémorragie rétiniennes, tortuosités des vaisseaux sanguins et malformations vasculaires;

3.) l'occlusion des capillaires rétiniens, une forme d'altération vasculaire, complication du diabète pouvant causer la cécité.

\section{Recommandations}

Chez les personnes de 15 ans et plus atteintes de diabète de type 1, un expert doit procéder au dépistage et à l'évaluation de la rétinopathie annuellement à compter de cinq ans après l'apparition du diabète.

Chez les personnes atteintes de diabète de type 2 , un expert doit procéder au dépistage et à l'évaluation de la rétinopathie diabétique au moment du diagnostic de diabète. L'intervalle entre les évaluations subséquentes doit être déterminé en fonction de la gravité de la rétinopathie. Chez les personnes qui ne présentent pas de rétinopathie ou qui présentent une rétinopathie minime, l'intervalle recommandé est de un à deux ans.

Le dépistage de la rétinopathie diabétique doit être effectué par des professionnels expérimentés soit en personne, soit par l'évaluation de photographies rétiniennes prises après dilatation des pupilles. 
Pour prévenir l'apparition et retarder l'évolution de la rétinopathie diabétique, les personnes diabétiques doivent être traitées de façon à optimiser le contrôler de la glycémie et la maitrise de la TA. En présence de taux de lipides anormaux, on doit considérer que le risque de rétinopathie est élevé.

Les patients atteints de rétinopathie diabétique menaçant la vue doivent être évalués par un ophtalmologiste généraliste ou un spécialiste de la rétine. Il faut envisager un traitement au laser ou une vitrectomie ou un traitement pharmacologique.
Il faut diriger les personnes ayant une déficience visuelle vers les services voulus en vue d'une évaluation de leur vision et d'une réadaptation.

Les optométristes, qui sont les cliniciens de première ligne des soins oculovisuels, peuvent faire une différence chez les gens atteints de diabète grâce aux soins oculovisuels qu'ils dispensent. Nous espérons que cette information et que le chapitre complet sur la rétinopathie dans les Lignes directrices de pratique clinique 2008 (qu'on peut trouver à l'adresse diabetes.ca) vous aideront à étendre les soins à vos patients atteints de diabète.

†Comité d'experts des Lignes directrices de pratique clinique de l'Association canadienne du diabète. Lignes directrices de pratique clinique 2008 de l'Association canadienne du diabète pour la prévention et le traitement du diabète au Canada, Can. J Diabetes, 2008, 32 (suppl. 1), S134-139.

\section{Urgent help is needed to give sight to 300,000 people.}

\section{Say Yestoday}

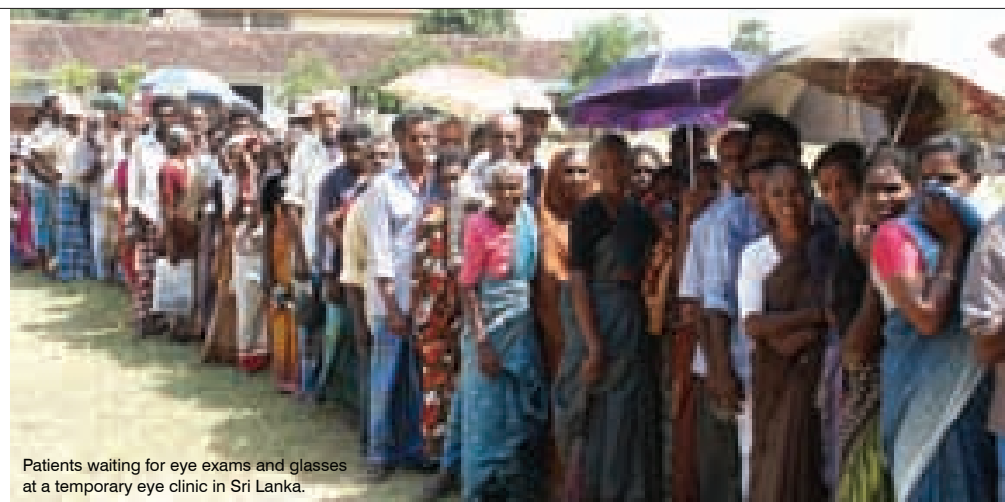

The Problem.

The Solution.

670 million people do not have access to the eye exams and glasses they need. Without glasses, children may be unable to learn at

Training local eye care professionals and establishing community school and parents unable to provide for their families. based vision centres and optical workshops are the only way to solve this global problem.

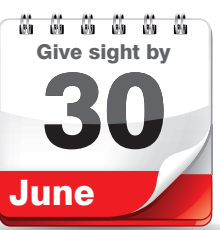

\section{Please help us give sight by June 30,} by making a donation today or by raising funds in your practice to:

- Establish 10 Vision Centres • Train 27 Optometric Professionals

- Equip 5 Optical Labs 